\title{
Coordenação do nado borboleta e estágio maturacional de jovens nadadores
}

http://dx.doi.org/10.11606/1807-5509201900030479

\section{Resumo}

0 objetivo deste estudo foi comparar a coordenação do nado borboleta de jovens nadadores em diferentes estágios maturacionais. Vinte e um nadadores entre 10 e 12 anos de idade divididos em dois grupos, de acordo com o estágio de maturação biológica em que se encontravam (EM2 - n=11 e EM3 - $n=10$ ) foram avaliados durante $25 \mathrm{~m}$ no nado borboleta em máxima intensidade. Os indicadores de coordenação entre as ações de braços e pernas, e o desempenho foram obtidos por meio de videogrametria e cronometragem, respectivamente. Resultados: Nadadores do grupo EM3 apresentaram melhores indicadores de coordenação e maior duração relativa das fases propulsivas da pernada. Os resultados apresentados indicam que jovens nadadores deveriam receber especial atenção com relação a indicadores coordenativos do nado borboleta, visando, especificamente, o sincronismo propulsivo entre o início da fase de empurrada da braçada e o início da fase descendente da segunda pernada.

Palavras-chave: Biomecânica; Parâmetros Coordenativos; Natação; Maturação Sexual.

\section{Introdução}

O nado borboleta é caracterizado por duas açóes simultâneas de pernas (em conjunto com a ondulação corporal) para cada ciclo completo de braçadas. A análise da coordenação desse nado possibilita estimar a duração das fases da pernada (duas açóes descendentes e duas ascendentes), da braçada (entrada e apoio, puxada, empurrada e recuperaçáo) e dos intervalos entre os momentoschave específicos da braçada e da pernada ${ }^{1}$. Desta forma, é possível avaliar a coordenação entre as açôes das pernas e dos braços e a continuidade propulsiva do nado ${ }^{1}$.

A coordenação entre braços e pernas no nado borboleta depende de restriçôes impostas à ação, que levam o nadador a uma auto-organização ótima². As restrições são divididas em ambientais, de tarefa e do organismo. As restrições ambientais estão associadas com fatores externos ao indivíduo. As restriçôes da tarefa descrevem a atividade a ser realizada e podem ser divididas em três categorias: (i) relativas ao objetivo, (ii) às regras ou instruçōes, e (iii) aos implementos (e.g. palmares). Finalmente, as restrições do organismo referem-se às características estruturais ou funcionais do indivíduo que, por sua vez, podem impactar a coordenação.

A maturação biológica é um processo que provoca alterações estruturais e funcionais no indivíduo ${ }^{3}$. Portanto, pode atuar como uma restrição do organismo sobre a coordenação do nado borboleta. Apesar de existir tanto um padrão quanto um cronograma para o processo de maturação, os indivíduos amadurecem em diferentes momentos e em diferentes taxas, especialmente durante a idade pré-púbere, que pode variar de 10-15 anos ${ }^{3}$. No
*Universidade Federal do Rio Grande do Sul, Porto Alegre, RS, Brasil.

**Faculdade de Ciências do Exercício e do Esporte, Universidade de Verona, Verona, Itália.

${ }^{* * *}$ Faculdade de Educação Física, Universidade Estadual de Campinas, Campinas, SP, Brasil.

${ }^{* * * *}$ Faculdade de Educação Física, Universidade Municipal de São Caetano do Sul, São Caetano do Sul, SP, Brasil.

***** Meazure Ciências do Esporte, São Paulo, $\mathrm{SP}$, Brasil. 
entanto, as competições e o treinamento de natação são geralmente divididos em grupos etários (por exemplo, 10-12 anos), o que sugere que, na mesma faixa etária, é possível haver indivíduos pré e póspúberes treinando e competindo juntos.

Uma vez que o estágio maturacional é uma restrição que pode afetar a coordenação do nado borboleta, o presente estudo tem como objetivo comparar a coordenação do nado borboleta de jovens nadadores em diferentes estágios maturacionais. Considerando que (1) a técnica é dependente de capacidade coordenativa e que, (2) em uma mesma categoria (petiz, 10-12 anos), atletas podem apresentar diferentes níveis maturacionais, estabeleceu-se, como hipótese, que nadadores com maturação mais adiantada apresentam melhores indicadores de coordenação no nado borboleta.

\section{Método}

\section{Amostra}

Participaram deste estudo 21 nadadores competitivos com idade entre 10 e 12 anos, sendo 11 meninos e 10 meninas. Os atletas participavam regularmente de competições e tinham, em média, 33,4 $\pm 13,5$ meses de experiência em treinamento de natação. No momento da coleta de dados, o treinamento desses nadadores era composto por, no mínimo, três sessões semanais que tinham duração aproximada de 60 minutos. Este projeto foi aprovado pelo Comitê de Ética em Pesquisa da universidade onde foi desenvolvido (número 21488), sendo que todos os participantes e seus responsáveis receberam e assinaram um termo de consentimento livre e esclarecido.

\section{Procedimentos experimentais}

Todos os testes foram realizados em um único dia em uma piscina de $25 \mathrm{~m}$ coberta e aquecida (temperatura da água $-29^{\circ} \mathrm{C}$ ). Como aquecimento os atletas realizaram de 400 a 600 m nado livre, em baixa intensidade, com exercícios de técnica do nado borboleta. Dez minutos após o fim do aquecimento, os nadadores realizaram um esforço máximo de 25 m partindo de dentro da piscina para avaliação do desempenho e da coordenação de nado. Após o final do teste de desempenho, os nadadores realizaram a auto-avaliação do estágio maturacional.

\section{Desempenho}

O desempenho foi determinado pelo tempo despendido no percurso da distância de $25 \mathrm{~m}$ utilizando o nado borboleta em intensidade máxima. A cronometragem foi realizada por três pesquisadores experientes de forma manual e considerou o intervalo decorrido entre a perda de contato do pé do atleta com a borda da piscina e o toque das suas mãos na borda oposta. O tempo médio dos três cronômetros foi retido para análise.

\section{Coordenação do nado borboleta}

A determinação dos momentos-chave para a análise da coordenação e da duração das fases da braçada e pernada foi realizada por meio de videogrametria subaquática durante o teste de desempenho. As imagens foram obtidas por uma câmera de vídeo subaquática (modelo: SANYO, VPC-WH1; frequência de amostragem: $60 \mathrm{~Hz}$ ), deslocada manualmente, por meio de um carrinho sobre trilhos na borda lateral da piscina ao longo de $10 \mathrm{~m}$ (dos 10 aos $20 \mathrm{~m}$ do percurso de 25 $\mathrm{m})$. Isso possibilitou a aquisição da perspectiva sagital do nado, cujas imagens foram analisadas posteriormente no software Kinovea 0.8 .15 para a determinação das seguintes variáveis ${ }^{1}$ :

(1) Duração das fases da braçada - fase de entrada e apoio: tempo decorrido entre o momento de entrada das mãos na água e o momento-chave que antecede o deslocamento para trás das mãos; fase de puxada: tempo decorrido entre o momentochave que antecede o movimento das mãos para trás até o momento em que as mãos estejam posicionadas verticalmente abaixo do ombro; fase de empurrada: tempo decorrido entre o momento em que as mãos estejam posicionadas verticalmente em relação aos ombros até o momento de saída das mãos da água; e fase de recuperação: tempo decorrido entre o momento de saída até o momento de entrada das mãos novamente na água. Essas fases foram expressas em percentual da duração de um ciclo de braçada. 
Dos $25 \mathrm{~m}$, entre os 10 e os $20 \mathrm{~m}$ do percurso, três ciclos foram analisados e a média foi retida. A duração das fases ditas propulsivas (puxada e empurrada) e não propulsivas (entrada e apoio, e recuperação) foram somadas, respectivamente em Prop e Nprop.

(2) Fase descendente 1 (D1): tempo decorrido entre o momento de mudança de direção superior e o momento de mudança de direção inferior da primeira pernada; fase descendente 2 (D2): tempo decorrido entre o momento de mudança de direção superior e o momento de mudança de direção inferior da segunda pernada; fase ascendente 1 (A1): tempo decorrido entre o momento de mudança de direção inferior e o momento de mudança de direção superior da primeira pernada; e fase ascendente 2 (A2): tempo decorrido entre o momento de mudança de direção inferior e o momento de mudança de direção superior da segunda pernada. Essas fases foram expressas em percentual da duração de um ciclo de pernada. A duração das fases ditas propulsivas (D1 e D2) e não propulsivas (A1 e A2) foram somadas, respectivamente em Prop e Nprop. Dos $25 \mathrm{~m}$, entre os 10 e os $20 \mathrm{~m}$ do percurso, três ciclos completos foram analisados e a média foi retida.

(3) Coordenação braçada-pernada: T1: diferença temporal entre o momento da entrada das mãos na água e o momento-chave inicial de D1; T2: diferença temporal entre o momento-chave de inicio da fase de puxada e o momento-chave final de D1; T3: diferença temporal entre o momento-chave de início da fase de empurrada e o momento-chave inicial de D2; e T4: diferença temporal entre o momento-chave de final da fase de empurrada e o momento-chave final de D2. Resultados expressos em percentual de duração de um ciclo de pernada. Dos $25 \mathrm{~m}$, entre os $10 \mathrm{e}$ os $20 \mathrm{~m}$ do percurso, três ciclos foram analisados e a média foi retida.

(4) Os valores de coordenação braçada-pernada em $\mathrm{T} 1, \mathrm{~T} 2$, T3 e T4 foram interpretados de acordo com ChOllet et al. ${ }^{1}$ :

- Quando T1=0: há continuidade entre o fim da fase de recuperação e o início da fase descendente (i.e. o início da propulsão);

- Quando T1>0: os braços iniciam a fase de entrada e apoio enquanto as pernas ainda se recuperam da fase ascendente (considerada não-propulsiva) do ciclo anterior. Portanto, os braços deslizam à frente até o momento em que as penas completam a fase ascendente. Assim, há um intervalo sem propulsão em que os braços deslizam à frente e é realizada a recuperação da segunda pernada;

- Quando T1<0: a propulsão da pernada inicia antes do fim da recuperação dos braços; isso corresponde a uma superposição de duas ações que são contraditórias (i.e. recuperação dos braços e propulsão das pernas). Essa condição não é recomendada pois as pernas propulsionam o corpo à frente sem que os braços estejam numa posição hidrodinamicamente favorável. De fato, a recuperação dos braços ocasiona uma posição mais vertical do tronco;

- Quando T2=0: há continuidade entre a ação das pernas e dos braços, isto é, não há intervalo sem propulsão;

- Quando T2>0: há sobreposição das ações propulsivas de pernas e braços. Os braços estão na fase de puxada e as pernas já iniciaram sua ação propulsiva;

- QuandoT2<0: Os braços deslizam passivamente enquanto as pernas completam sua ação ascendente (considerada não-propulsiva). Há um intervalo sem propulsão na posição de deslize.

- Quando T3=0: há sincronismo entre o início da fase de empurrada e o inicio da fase descendente da segunda pernada.

- Quando T3>0: a fase descendente da segunda pernada inicia após o inicio da fase de empurrada.

- Quando T3<0: há falta de sincronismo, que corresponde a fase descendente da segunda pernada iniciando antes do início da fase de empurrada da braçada.

- Quando T4=0: o momento-chave final da segunda pernada ocorre simultaneamente com a saída das mãos da água, isto é, a fase descendente propulsiva da ondulação facilita a recuperação dos braços;

- Quando T4>0: as mãos saem da água antes do final da fase descendente da segunda pernada. Isso corresponde a uma sobreposição de duas ações que são contraditórias, pois a posição do corpo durante a fase de recuperação da braçada não oferece vantagem à propulsão de pernas;

- Quando T4<0: a fase descendente da segunda pernada termina antes do final da fase de empurrada.

Já o somatório dos módulos das durações 
relativas, ou intervalo temporal total (TTG), foi considerado um indicador da coordenação, pois um menor TTG reflete uma maior continuidade entre as ações propulsivas de braços e pernas ${ }^{1}$.

\section{Maturação Biológica}

O indicador sexual secundário obtido com o protocolo de auto avaliação de MATsudo e MATSUDO $^{4}$ foi utilizado para classificar os indivíduos de acordo com os seguintes elementos: 1) pilosidade púbica (ambos os sexos); 2) características genitais (exclusivo sexo masculino); 3) características de mamas (exclusivo sexo feminino). Foi realizada uma inspeção única e exclusivamente visual das características citadas anteriormente. Essa inspeção foi realizada pelo próprio indivíduo sem roupa e colocado frente a um espelho. Dessa forma comparou-se sua imagem no espelho com as imagens-referências ${ }^{5}$. A partir da inspeção e comparação com as imagens é atribuída uma pontuação, em uma escala sem números decimais, que varia de 1 a 6 .

\section{Resultados}

Os grupos EM2 ( $\mathrm{n}=10$, sete meninos e três meninas) e EM3 ( $\mathrm{n}=11$, quatro meninos e sete meninas) apresentaram, respectivamente, 11,2 $\pm 0,8$ e $11,6 \pm 0,7$ anos de idade; $36,1 \pm 16,4$ e 30,0 $\pm 10,2$ meses de prática; e $19,7 \pm 2,8$ e $19,5 \pm 2,1 \mathrm{~s}$ de desempenho nos $25 \mathrm{~m}$ nado borboleta. Nenhuma diferença foi identificada entre os grupos maturacionais para idade, tempo de prática/treinamento na modalidade

\section{Análise Estatística}

A análise estatística contou, primeiramente, com a aplicação do teste de Shapiro Wilk para verificação da normalidade dos dados. Após, foi realizado o cálculo das medidas de tendência central e dispersão das variáveis quantitativas (i.e. média e desvio padrão). Do total de participantes, 10 reportaram estar no estágio maturacional 2, enquanto 11 encontravam-se no estágio 3. Assim, foram criados dois grupos de maturação (EM2 e EM3). A comparação de todas as variáveis entre os grupos de maturação foi realizada com a aplicação de um teste t de Student para dados independentes. O nível de significância foi estabelecido em 0,05. Os tamanhos de efeito foram identificados apenas quando diferenças foram constatadas, com a estatística d de Cohen utilizando-se as médias e desvios-padrão, sendo critérios de classificação os propostos por $\mathrm{HopKINS}^{6}$ : de 0 a 0,2 : trivial; de 0,21 a 0,6 : pequeno; de 0,61 a 1,2: moderado; de 1,21 a 2,0: grande e maior que 2,0: muito grande.

TABELA 1 -Média \pm DP da duração relativa das fases de braçada e de pernada. Comparações entre os grupos de maturação (EM2 e EM3).

\footnotetext{
a diferença para $\operatorname{EM} 2\left(\mathrm{t}_{19}=-2,79\right.$; $\mathrm{p}=0,011 ; \mathrm{d}$ de Cohen =1,2);

b diferença para $\operatorname{EM} 2\left(\mathrm{t}_{19}=-2,17\right.$; $\mathrm{p}=0,042 ; \mathrm{d}$ de Cohen = 0,99); c diferença para $\operatorname{EM} 2\left(\mathrm{t}_{19}=2,25\right.$; $\mathrm{p}=0,036 ; \mathrm{d}$ de Cohen $=1,32$ ).
}

\begin{tabular}{ccc}
\hline & EM2 $(\mathbf{n}=10)$ & EM3 $(\mathbf{n}=\mathbf{1 1})$ \\
\hline Fases da Braçada & & \\
Entrada e Apoio (\%) & $36,9 \pm 8,9$ & $34,6 \pm 7,5$ \\
Puxada (\%) & $23,8 \pm 4,7$ & $24,6 \pm 4,5$ \\
Empurrada (\%) & $17,3 \pm 3,6$ & $15,8 \pm 2,9$ \\
Recuperação (\%) & $22,0 \pm 5,3$ & $25,0 \pm 3,2$ \\
Prop (\%) & $41,1 \pm 5,2$ & $40,4 \pm 5,6$ \\
N-prop (\%) & $58,9 \pm 5,2$ & $59,6 \pm 5,6$ \\
\hline
\end{tabular}

continua 
continuação

\begin{tabular}{ccc}
\hline & EM2 $(\mathbf{n}=\mathbf{1 0})$ & EM3 $(\mathbf{n}=\mathbf{1 1})$ \\
\hline Fases da Pernada & & \\
D1 $(\%)$ & $16,1 \pm 1,8$ & $18,3 \pm 2,1^{\text {a }}$ \\
A1 $(\%)$ & $33,6 \pm 3,5$ & $34,1 \pm 5,1$ \\
D2 $(\%)$ & $15,5 \pm 1,9$ & $17,8 \pm 1,8^{\mathrm{b}}$ \\
A2 $(\%)$ & $34,8 \pm 5,4$ & $29,8 \pm 5,2^{\mathrm{c}}$ \\
\hline
\end{tabular}

No tocante aos dados de coordenação braçadapernada (TABELA 2), pode-se observar que, em termos percentuais, o grupo EM3 apresenta menores valores para T2, T3 e T4. Porém diferença significativa é encontrada para TTG, ou seja, o grupo EM3 apresenta maior continuidade propulsiva do que o grupo EM2, o que indica melhor coordenação de nado.

TABELA 2 -Média \pm DP dos intervalos braçada-pernada. Comparações entre os grupos de maturação (EM2 e EM3).

\begin{tabular}{ccc}
\hline & EM2 $(\mathbf{n = 1 0})$ & EM3 $(\mathbf{n}=\mathbf{1 1})$ \\
\hline T1 $(\%)$ & $-0,4 \pm 6$ & $-0,5 \pm 2$ \\
T2 $(\%)$ & $-21 \pm 8$ & $-16 \pm 7$ \\
T3 $(\%)$ & $-11 \pm 8$ & $-7 \pm 5$ \\
T4 $(\%)$ & $-13 \pm 9$ & $-5 \pm 6$ \\
TTG $(\%)$ & $45,4 \pm 26$ & $28,5 \pm 15^{\mathrm{a}}$ \\
\hline
\end{tabular}

a diferença para $\operatorname{EM} 2\left(t_{19}=2,14\right.$; $\mathrm{p}=0,04 ; \mathrm{d}$ de Cohen $=0,90)$.

\section{Discussão}

O presente estudo comparou a coordenação do nado borboleta de nadadores de 10 a 12 anos em diferentes estágios maturacionais. Nossos dados apontam que as prováveis modificações inerentes ao avanço dos estágios maturacionais parecem influenciar a forma com que os atletas coordenam as ações propulsivas do nado borboleta. De fato, o intervalo total (TTG) encontrado nos atletas em estágio maturacional mais avançado foi consideravelmente menor, indicando maior continuidade propulsiva do nado. Essa maior continuidade propulsiva parece ser consequência principalmente da redução dos intervalos T3 e T4. Estes resultados confirmam a hipótese formulada (nadadores com maturação mais adiantada apresentam melhores indicadores de coordenação no nado borboleta). Cabe ressaltar que os tamanhos de efeito encontrados para as duraçóes de D1, D2, A2 e TTG situaram-se entre moderados e grandes, com semelhantes magnitudes.

O intervalo T3 observado foi negativo em ambos os grupos, indicando que a propulsão das pernas (fase descendente da segunda pernada) iniciou antes do término da fase de puxada dos braços. Estudos prévios $^{1,7,8}$ que avaliaram atletas adultos ( -20 anos) e com desempenho superior (velocidades entre $1,63-1,74 \mathrm{~m} / \mathrm{s} \times-1.28 \mathrm{~m} / \mathrm{s}$ no presente estudo) reportaram valores maiores de duração para a fase D2 da pernada $(-22-26 \%)$ do que os encontrados no presente estudo $(-15-17 \%)$. Assim, o aumento da duração relativa da fase descendente na segunda ondulação parece estar relacionado ao processo de maturação, ao passo que tempo de prática não foi diferente entre EM2 e EM3.

Os valores de T4 (cerca de -5\%) apresentados pelos nadadores do grupo EM3 são próximos aos resultados de estudos anteriores, que verificaram valores entre -3 e $2 \%{ }^{1,8}$, diferentemente do grupo EM2 em que a descontinuidade foi maior (cerca de $-13 \%)$. Como esse intervalo negativo está associado ao tempo entre o final da fase descendente $\mathrm{da}$ segunda pernada e o final da fase de empurrada da braçada (mãos saindo da água antes do final da pernada), uma melhor execução da fase descendente da segunda pernada deve reduzir esse intervalo.

A maior continuidade propulsiva identificada 
no grupo EM3 sugere que com o avanço da maturação o sincronismo entre momentos-chave das açôes propulsivas de perna e braço melhora, indicando uma coordenação superior. Essa melhor sincronia, especialmente nos momentos T3 e T4, é um forte indicativo de que o aperfeiçoamento da fase descendente da segunda pernada exerce um papel determinante na melhora da coordenação e da continuidade propulsiva no nado borboleta. Desta forma, treinadores deveriam desenvolver e prescrever, desde as categorias inferiores, exercícios de técnica focando (1) na finalização da propulsão da segunda pernada simultaneamente à finalização da fase de empurrada da braçada, para obter um maior sincronismo de T4, e (2) no início da fase de empurrada da braçada simultaneamente ao inicio da fase propulsiva da segunda pernada, para obter um maior sincronismo de T3.

Interessantemente, o desempenho dos grupos foi semelhante mesmo havendo diferença na coordenação e na continuidade propulsiva. Sabendo da grande importância da técnica e da coordenação para o desempenho na natação, em que a resistência oferecida pelo meio aumenta ao quadrado do aumento da velocidade de nado' ${ }^{9}$, é desejável que a melhora na coordenação preceda o treinamento de força no processo de formação esportiva dos sujeitos. Em corroboração, HaYRILUK ${ }^{10}$ comparou a força da mão durante o nado e também a técnica (por meio do coeficiente de arrasto) de dois grupos de nadadores de borboleta de diferentes níveis $(1,56 \mathrm{~m} / \mathrm{s} \times 1,37 \mathrm{~m} / \mathrm{s})$. A diferença entre os grupos apresentou tamanhos de efeito de 0.25 (pequeno) para a força e de 1,17 (moderado) para a técnica, indicando que a vantagem dos nadadores mais rápidos deriva mais da técnica do que da força.

Além disso, é também possível sugerir que as diferenças encontradas na coordenação influenciem mais o desempenho em distâncias mais longas. $\mathrm{O}$ aumento da continuidade propulsiva pode representar menores variaçôes intracíclicas da velocidade e menor custo energético ${ }^{11}$. $\mathrm{O}$ menor custo energético para uma dada velocidade média significa a possibilidade de maior economia de substratos energéticos e menores alterações na homeostase intracelular, consequentemente promovendo o aumento da velocidade e melhora do desempenho em distâncias superiores a $25 \mathrm{~m}$.

É importante considerar que a utilização da auto avaliação dos indicadores sexuais secundários contém limitaçôes quanto ao entendimento dos componentes a serem analisados para gerar a classificação e, consequentemente, ocasionar eventual superestimação do estágio maturacional ${ }^{12}$. Por outro lado, entendemos que esse método se mostra como uma alternativa em estudos que não haja um médico capacitado ou apropriado para aplicar a classificação de maturação biológica via indicador sexual secundário.

Finalmente, a maturação dos nadadores em idade pré-púbere parece ser um fator importante a ser considerado na coordenação do nado borboleta, apesar de não afetar o desempenho máximo em curtas distâncias.

\title{
Agradecimentos
}

Aos atletas que participaram deste estudo e a todos que se disponibilizaram para auxiliar na realização do mesmo.

\begin{abstract}
Butterfly coordination and maturational stage of young swimmers

The aim of this study was to compare the butterfly stroke coordination in young swimmers at different maturational stages. Twenty-one swimmers aged from 10 to 12 years, divided into two groups, according to the biological stage of maturation (SM2 $-n=11$ and SM3 $-n=10$ ) were evaluated during $25 \mathrm{~m}$ in butterfly stroke in maximum intensity. Coordination indicators between the actions of arms and legs, and performance were obtained through videogrammetry and timing, respectively. Swimmers in SM3 showed better indicators of coordination and greater relative duration of the propulsive phases of the kick. The results indicate that young swimmers should receive special attention in relation to coordinative indica-
\end{abstract}


tors, aiming specifically, the propulsive timing between the start of the pull phase of the stroke and the beginning of the descending phase of the second kick.

KEYwoRDs: Biomechanics; Coordinative Parameters; Swimming; Sexual Maturation.

\section{Referências}

1. Chollet D, Seifert L, Boulesteix L, Carter M. Arm to leg coordination in elite butterfly swimmers. Int J Sports Med. 2006;27(4):322-9.

2. Newell KM. Constraints on the development of coordination. In: Motor development in children: aspect of coordination and control. In: Wade MG, Whiting HTA, editores. Dordrecht: Nijhoff; 1986. p.341-360.

3. Malina RM, Bouchard C, Bar-Or O. Growth, maturation, and physical activity. 2a ed. Champaign: Human kinetics; 2004.

4. Matsudo SMM, Matsudo VKR. Validade da auto avaliação na determinação da maturação sexual. Rev Bras Ciência Mov. 1991;5(2):18-35.

5. Tanner JM. Growth at adolescence. Oxford: Blackwell Scientific; 1962.

6. Hopkins WG. Measures of reliability in sports medicine and science. Sports Med. 2000;30(1):1-15.

7. Seifert L, Boulesteix L, Chollet D, Vilas-Boas JP. Differences in spatial-temporal parameters and arm-leg coordination in butterfly stroke as a function of race pace, skill and gender. Hum Mov Sci. 2008;27(1):96-111.

8. Telles T, Barroso R, Barbosa AC. Effect of hand paddles and parachute on butterfly coordination. J Sports Sci. 2015;33(10):1084-92.

9. Toussaint HM, Hollander P, Berg C, Vorontsov AR. Biomechanics of Swimming. In: Garret WE, Kirkendall DT, organizadores. Exercise and Sport Science. Philadelphia: Lippincot Willians and Wilkin; 2000. p. 639-659.

10. Havriluk R. Performance Level Differences in Swimming: Relative Contributions of Strength and Technique. XIth International Symposium for Biomechanics and Medicine Swimming; 16-19 jun. 2010; Oslo. Oslo: Biomechanics and Medicine Swimming XI; 2010. p. 321-323.

11. Barbosa TM, Keskinen KL, Fernandes R, Colaço P, Lima AB, Vilas-Boas JP. Energy cost and intracyclic variation of the velocity of the centre of mass in butterfly stroke. Eur J Appl Physiol. 2005;93(5-6):519-23.

12. Rasmussen AR, Wolfhart-Veje C, Tefre de Renzy-Martin K, Hagen CP, Tinggaard J, Mouritsen A, et al. Validity of self-assessment of pubertal maturation. Pediatrics. 2015;135(1):86-93.

ENDERECOO DE CORRESPONDÊNCIA:

Marcos Franken

Avenida Paulo Gama, 750 - Farroupilha

Porto Alegre - RS - BRASIL

CEP: 90040-060

E-mail: marcos_franken@yahoo.com.br
Submetido: 04/07/2016

Aceito: 20/09/2017 